\title{
Essais
}

ESSAIS

Revue interdisciplinaire d'Humanités

5 | 2014

Médias et élites

\section{L'écriture de la dénégation à propos de Cesare Battisti, Face au mur}

Laura Lauri

\section{(2) OpenEdition}

1 Journals

Édition électronique

URL : http://journals.openedition.org/essais/8257

DOI : 10.4000/essais. 8257

ISSN : 2276-0970

Éditeur

École doctorale Montaigne Humanités

Édition imprimée

Date de publication : 15 juillet 2014

Pagination : 110-125

ISBN : 978-2-9544269-3-8

ISSN : 2417-4211

\section{Référence électronique}

Laura Lauri, «L'écriture de la dénégation à propos de Cesare Battisti, Face au mur », Essais [En ligne], 5 | 2014, mis en ligne le 13 avril 2021, consulté le 15 avril 2021. URL : http://journals.openedition.org/ essais/8257 ; DOI : https://doi.org/10.4000/essais.8257 


\section{L'écriture de la dénégation à propos de Cesare Battisti, Face au mur}

\section{Laura Lauri}

Quand le roman de Cesare Battisti ${ }^{1}$ Face au mur sort aux éditions Flammarion, le 7 mars 2012, certains des journaux français lui consacrent quelques lignes, mais presque tous semblent porter leur attention plutôt sur son auteur, ex-militant italien de l'extrême gauche, que sur l'ouvrage lui-même.

Cette contribution a comme but l'étude de l'écriture de ce roman dans une perspective plus vaste visant aussi les enjeux politiques qui se dissimulent derrière les aspects purement littéraires. Elle s'insère dans un plus vaste travail de recherche consacré à toute l'œuvre littéraire de Cesare Battisti ainsi qu'au rôle que ce personnage a acquis en Italie et en France, à travers les nombreuses

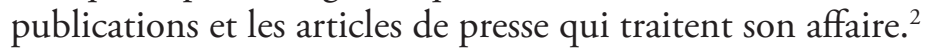

1 Cesare Battisti est un ressortissant italien ayant fait partie des Prolétaires Armés pour le Communisme (PAC) pendant les années de plomb italiennes. Il a été condamné par contumace pour quatre assassinats commis entre 1978 et 1979. Il s'est évadé de prison en 1982 avec l'aide de ses compagnons d'armes et s'est exilé au Mexique jusqu'en 1990. Grâce à la doctrine Mitterrand, il s'est ensuite réfugié en France où il est devenu écrivain et a publié plusieurs romans policiers. En 2004, il s'est enfui de France car le gouvernement français a accepté d'extrader les terroristes italiens. Il se rend alors au Brésil, où il est arrêté en 2007. Le Président Luis Inàcio Lula da Silva décide en 2011 de le libérer et de lui accorder l'asile. Cesare Battisti vit actuellement au Brésil.

2 En Italie : Evangelisti Valerio, Genna Giuseppe, Ming 1 Wu, Il caso Battisti, Città di Castello (PG), NdA press, 2004 ; Cruciani Giuseppe, Gli amici del terrorista, Cles (TN), Sperling \& Kupfer Editori, 2010 ; Galli Giorgio, Dossier Cesare Battisti, Bollate, Kaos Edizioni, 2011 ; Turone Giuliano, Il caso Battisti, Milano, Garzanti, 2011 ; Bocca Giorgio, «Rivoluzione e voglia di denaro. Storia di un bandito a Milano », La Repubblica, 19/09/1985; Marinetti Cesare, "I paradossi di Battisti che non nega i fatti ma il processo ", La Stampa, 15/07/2004 ; Bianconi Giovanni, "Non dica però di essere innocente ", Corriere della Sera, 31/12/2010 ; Manzo Paolo, "Intervista a Edoardo Suplicy, il grande difensore di Battisti in Brasile ", Panorama, 5/01/2011.

En France : Vargas Fred, La vérité sur Cesare Battisti, Paris, Viviane Hamy éditions, 2004 ; Perrault Guillaume, Génération Battisti, ils ne voulaient pas savoir, Paris, Plon, 2005 ; de Luca Erri, "Vengeance infinie ", Le Monde, 22/02/2004; Pennac Daniel, "Lettre à Cesare Battisti », Le Monde, 24/02/2004 ; Lévy Bernard-Henri, « Bloc-notes », Le Point, 11/03/2004; 
Il y a donc un écart entre l'abondance d'ouvrages et d'articles de presse consacrés à l'Affaire Battisti et la pénurie des analyses de son écriture.

En outre, en Italie, il est difficile de se procurer les livres de Cesare Battisti. Sur 14 ouvrages de cet auteur, seulement 4 sont encore publiés, dont deux par les éditions DeriveApprodi, qui sur leur site internet annoncent : «DeriveApprodi è una casa editrice indipendente. Si occupa di agitazione culturale e politica $»^{3}$. Les livres encore publiés sont : Avenida Revolucion aux éditions Nuovi Mondi qui relate la vie près du mur séparant le Mexique des États-Unis, Luultimo sparo. Un "delinquente comune" nella guerriglia italiana, traduit en français Dernières cartouches aux éditions DeriveApprodi qui raconte l'échec et la fin de la lutte armée, L'orma rossa, traduit en français L'ombre rouge, aux éditions Einaudi, qui traite du cas des ressortissants italiens des années de plomb vivant dans une société française qui les ignore et $A u$ pied du mur publié par Flammarion, en français, et traduit en italien aux éditions Brossura. Cesare Battisti a, en effet, écrit son dernier ouvrage en langue française. Sur la base de ces données, on pourrait se demander si Cesare Battisti n'est pas condamné, en Italie, à une sorte de damnatio memoriae en ce qui concerne au moins une grande partie de son œuvre. Plusieurs de ses romans, en effet, ne sont plus disponibles en langue italienne, mais désormais seulement en traduction.

Lécriture du roman Face au mur est engendrée par l'enfermement au sein d'un pénitencier et jaillit d'une culture nourrie de lectures d'auteurs classiques. Cependant, dans cette œuvre, il n'y a pas que des réminiscences littéraires fortuites mais plutôt une série de citations tirées d'une hypothétique bibliothèque d'auteurs choisis par le narrateur, dans un but bien précis. Dans cet univers carcéral, le lecteur est conduit vers une réflexion sur le thème de la punition par la réclusion et vers une plus vaste spéculation sur la responsabilité de la société dans certains crimes commis.

Tout au long du roman, la réalité se scinde en deux, dans un dualisme à l'allure manichéiste qui façonne un monde où les personnages féminins, en général, sont enfermés dans leur opacité aux abîmes sombres, tandis que les personnages masculins bénéficient toujours d'un jugement positif. Une écriture de la dénégation cherche à nier les faits, à les dissimuler afin de pouvoir mieux les dompter. C'est un vaste travail psychanalytique que d'accepter ce qui est désormais inacceptable. Alors, l'écriture devient capable d'éclairer les profondeurs les plus sombres de l'esprit et de créer des mondes infinis bien qu'à l'intérieur des murs d'une prison. L'écriture, dans ce roman, acquiert les fonctions d'une

Bouniot Sophie, «Le gouvernement trahit la parole de la France », "l'Humanité», 16/02/2009; Lévy Bernard-Henri, "Lettre ouverte au Président Lula sur le cas Battisti », Le Point, 3/12/2009. Cette liste est loin d'être exhaustive tant l'Affaire Battisti a fait couler d'encre.

3 DeriveApprodi est une maison d'éditions indépendante qui s'occupe d'agitation culturelle et politique. Traduction par nos soins. 
reconstruction sur les décombres de vies dévastées et se relève alors démiurge d'un monde qui, loin d'être parfait, en devient tout de même tolérable.

\section{Les éléments poïétiques donnant naissance à une écriture « savante »}

Le roman Face au mur, publié chez Flammarion en 2012 raconte l'histoire d'Auguste, enfermé dans une prison de Brasilia à cause de son passé de terroriste pendant les années 1970 en Italie. Il redoute l'extradition vers son pays natal. Il suit, chaque jour, le va-et-vient d'un petit oiseau, pendant la promenade qui lui est octroyée dans la cour du pénitencier.

C'est dans cet univers carcéral qu'il arrive à pouvoir se livrer à une véritable introspection afin d'analyser ses états d'âme tout en reconsidérant son parcours de combattant désabusé. Dans cette prison, Auguste décide enfin de se regarder en face et cette découverte de soi le mène à l'étude des autres : soit ceux qui sont au-delà des murs, visités à la lumière du souvenir, soit ceux qui sont en deçà des remparts, à savoir les prisonniers, décrits avec une plume teintée d'une touchante humanité.

On dirait alors que l'enfermement est le gage de la connaissance de l'essence ultime des choses : "Depuis que je suis privé de glace " dit le protagoniste "j’ai appris à me regarder sans crainte de me voir ${ }^{4}{ }^{4}$

C'est le va-et-vient d'un petit oiseau, entre une antenne sur le toit d'un immeuble et la branche d'un eucalyptus qui se trouve à l'intérieur de la cour du pénitencier, qui attire l'attention d'Auguste. À l'intérieur des murs qui enferment Auguste, le manège de l'oiselet incite, en quelque sorte, le personnage à laisser errer ses pensées et ses souvenirs entre son passé et son présent et donc entre sa vie en dehors de cette prison et son existence actuelle.

Alors, à l'instar du poisson qui vécut sur les rives du Tibre, dans la satura $\mathrm{V}^{5}$ de Juvénal, capable de remonter les conduits jusqu'en pleine Subure, Auguste s'immisce dans les récits des existences des prisonniers, ses compagnons d'infortune.

Et comme les imposants murs de la Subure endiguaient, dans la Rome antique, la pauvreté et le crime, ainsi les remparts de la prison d'Auguste

4 Battisti Cesare, Face au mur, Paris, Flammarion, 2012, p. 13.

5 Vos anguilla manet longae cognata colubrael Aut glacie aspersus maculis Tiberinus et ipsel Vernula riparum, pinguis torrente cloacal E tsolitus mediae cryptam penetrare Suburae. Juvénal, Satura V, p. 103-106. "Pour vous, on a réservé une anguille parente de la couleuvre effilée ou un poisson tacheté par la glace, qui vécut sur les rives du Tibre, qui s'est engraissé dans les flots des égouts et qui avait l'habitude de remonter leur conduits jusqu'en pleine Subure. "Traduction par nos soins. La comparaison entre la Subure et la prison d'Auguste semble convenir car dans l'œuvre de Cesare Battisti le thème du " mur » revient constamment et la Subure est l'archétype du rempart construit pour contenir l'indigence et la délinquance. 
semblent enfermer l'indigence et les délits de ces repris de justice ayant perdu le droit de vivre hors de ces enceintes.

L'incipit de Face au mur s'inscrit dans la tradition littéraire la plus classique à travers cet expédient à première vue futile : l'oiselet et son manège. En effet, on peut évoquer l'affairement frénétique de l'oiseau décrit par Corrado Govoni dans sa poésie Lo scricciolo ou encore citer les vers d'Eugenio Montale : « $i$ deliziosi figli della rugginel gli scriccioli o reatini come spessol li citano i poeti. "

Il est donc évident que l'idée de confier à un passereau le soin d'accompagner le flux de la pensée du protagoniste est un " topos " littéraire des plus fréquentés. Et comme le petit oiseau va de l'antenne à la branche d'eucalyptus et donc de l'extérieur de la prison à l'intérieur et vice-versa, ainsi le protagoniste alterne le souvenir du monde externe avec la description des détenus, en imitant, en quelque sorte, le va-et-vient du passereau. La particularité de cet oiseau réside dans le fait qu'il ne chante jamais. Ainsi le protagoniste remplacet-il ce chant par son récit.

Et si le manège de l'oiselet est un expédient littéraire, présent dans la littérature classique en tant qu'élément poétique et poïétique, les citations de différents auteurs, tout au long du roman, ont un but précis. La nouvelle de la Giara de Pirandello, citée par le protagoniste, offre une éloquente illustration d'une situation d'enferment psychologique : "Pour mon inefficacité à faire face à la vie réduite que javais cousue tout autour de moi ? Tel cet idiot dans "La Giara" de Pirandello, - comment s'en sortait-il déjà ? Casser "La Giara" et recommencer tout du début ? " Ici, la référence littéraire est surtout un expédient pour créer un raccourci qui guide le lecteur dans l'imagination de la coercition imposée au protagoniste du roman, dans la même situation grotesque que celle où se trouve le personnage de la nouvelle de Pirandello.

On reconnait ainsi les paroles de Marx dans la phrase que Cruel, un des détenus, adresse au protagoniste : "Je me demande ce que tu as contre le foot. Fais pas chier avec ce truc de la "drogue du peuple" [...] Oui, monsieur le philosophe de mes deux, la rage, parce que vous, les Italiens, vous ne digérez pas vos défaites contre nous. " ${ }^{8}$ Il est évident que l'adaptation ironique de la pensée de Marx au football n'est pas aussi intéressante que la reconnaissance d'une prééminence intellectuelle qu'Auguste posséderait au sein de ce monde carcéral.

6 Montale Eugenio, Al mare (o quasi), dans Quaderno dei quattro anni, Milano, Mondadori, I Meridiani, 1975, p. 620.

Traduction : "Ces délicieux enfants de la rouillel les troglodytes ou roitelets que bien souventl les poètes ont cités. " Dyerval Angelini Patrice, À la mer (en quelque sorte), in Cahier de poésie, 19731977, Paris, Gallimard, 1991, p. 280.

$7 \quad$ Face au mur, op. cit., p. 171.

8 Ibid., p. 104-105. 
Les reclus considèrent Auguste, bien que sarcastiquement, tout de même comme un "philosophe ", dépositaire d'un certain savoir que celui-ci semble dispenser à ses compagnons d'infortune. On remarque, en outre, que le registre de langue utilisé par les détenus est fort trivial. Le protagoniste, en revanche, se distingue, dans cette prison, par une langue soignée et correcte. Auguste est donc considéré par les autres captifs comme un détenu "savant " capable, entre autres, de citer Marx. Cela renforce la suprématie du protagoniste dans l'enceinte du pénitencier et, en même temps, confère une certaine autorité littéraire à son récit. On découvre le même mécanisme dans la citation de Sartre : "Tout projet existentiel de l'homme est destiné au fracas, parce que les possibilités qu'il évoque se montrent en dernière analyse impossibles à réaliser "?. Les mots de Sartre viennent clore une réflexion sur les années de combat du militant Auguste devenu terroriste. Il est vrai que cette citation, cette fois, ne s'adresse pas à d'autres personnages du récit dans un but pédagogique, mais directement au lecteur qui se voit contraint d'en recevoir la leçon.

Il est évident que de par ces citations, le protagoniste cherche à soutenir l'édifice de son écriture grâce à la littérature, tout en essayant d'asseoir son autorité d'intellectuel auprès du lecteur. Ainsi son étalage d'érudition littéraire sert-il à conquérir son statut de lettré s'immisçant dans la cour des élus, des intouchables, des intellectuels afin d'en recevoir le soutien. Une sorte d'immunité naîtrait donc de ce statut tout en se propageant par-delà l'écriture jusqu'à l'écrivain. Alors, même le prénom choisi pour le protagoniste semble désigner cette volonté : Augustus, à savoir " celui qui est majestueux, vénérable, consacré... auguste ».

\section{Une société corruptrice dans un monde scindé par des murs, entre manichéisme et perversion féminine}

Auguste trace le portrait des détenus qui l'entourent avec une technique qui relève du cinéma. Les souvenirs offrent souvent l'occasion, par analogie ou par contraste, de fixer le regard sur un des prisonniers afin d'en raconter l'histoire, à la manière d'un montage "attraction ", le procédé conçu par Eisenstein afin qu'une image se relie à l'autre par un lien logique. ${ }^{10}$

La constante qui réunit tous les personnages, sous ces cieux brésiliens, est une touchante humanité qui anime, de la même façon, du plus généreux des philanthropes jusqu'au plus impitoyable des criminels. Le mal qui ronge la

9 Face au mur, op. cit., p. 185.

10 Il s'agit d'une technique de montage d'images qui n'ont pas une relation immédiatement directe entre elles, mais que le spectateur arrive à relier par un lien logique, parfois métonymique, de la cause pour l'effet ou de comparaison, d'analogie, etc. Cf. Pinel Vincent, Le montage l'espace et le temps du film, Farignano, Cahiers du Cinéma, 2001, p. 61-87 et Rondolino Gianni, Tommasi Dario, Manuale del film, linguaggio racconto analisi, Torino, UTET, 1995, p. 157-159. 
société serait, selon le protagoniste, le pouvoir. Il faut se méfier du pouvoir, semble vouloir enseigner Auguste, à travers sa galerie de portraits. Dans ce roman, le pouvoir serait le responsable de la corruption du genre humain. On découvre là une certaine conception rousseauiste de la condition de l'homme qui naît avec une inclination naturelle à la bonté mais qui est amené, tout au long de sa vie, vers la corruption, par les institutions de la société où il lui faut vivre. En effet, Auguste semble adhérer, par ses " portraits " de détenus, à la théorie du philosophe des Lumières, exposée dans L'Émile, en démontrant que la faille de l'homme ne réside pas dans sa nature mais plutôt dans l'influence d'une société pernicieuse, délétère et dépravante. À partir de ce raisonnement, Auguste suggère, à mots couverts, que le détenu n'est pas le seul responsable de son crime. Ce concept soutient toute l'architecture du roman. Auguste est ce terroriste italien des années de plomb et alors le lecteur est amené à insérer Face au mur dans une perspective qui contemple, par-delà le romanesque, l'Histoire et les sciences sociales. Le narrateur, en effet, s'acharne à démontrer l'action corruptrice de la société sur chaque personnage décrit. Le lecteur est contraint à la réflexion sur les modalités de la punition du crime et même sur la légitimité de ce mur qu'Auguste fixe pendant l'heure de la promenade. On ne peut ne pas songer à l'essai Surveiller et punir où Michel Foucault analyse les institutions répressives de nos sociétés; ce dernier s'interroge sur la punition des crimes, sur l'organisation de la prison, voire sur son architecture, en citant Abel Blouet qui assure que "les murs sont la punition du crime» et encore que "les murs sont terribles, l'homme est bon ${ }^{11}$. Il est évident que par les considérations sur la légitimité de l'enfermement carcéral et sur la violence des gardes envers les détenus lors des fouilles, le protagoniste de Face au mur propose une vaste réflexion sur la légalité de l'enfermement de la part de l'État et sur les méthodes adoptées au sein des prisons. La violence des gardes est décrite, illustrée, savamment décomposée, non sans quelques touches ironiques, de la part du protagoniste lors des fouilles corporelles, afin que le lecteur éprouve le sentiment d'injustice face à ces actes : "Avant toute tentative de réaction $d u$ reclus, le bloc est investi par les agents: uniformes noirs, lunettes noires, matraques noires, fusils à pompe noirs, bombes à gaz noires [...]. Dans l'espace exigu vibrent les ordres criés par des gorges bien arrosées. Histoire de se donner du courage avant de braquer la quarantaine de détenus affaissés par groupes derrière l'acier de plusieurs grilles et qui, prudence oblige, pourraient encore opposer de la résistance à coups d'ongles et de dents. [...] nous sommes tous à poil, assis à même le sol, face aux WC, la tête baissée, les jambes écartées, chacun bien collé au derrière de l'autre: "On se bouge le cul, les connards; serrez-vous, les connards; [...] le premier qui lève les yeux de ses couilles, sìl en a, on lui ouvre le crâne, connards."

11 Blouet Abel, Projet de prison cellulaire pour 585 condamnés, précédé d'Observations sur le système pénitentiaire, Paris, Didot Frères, 1843, p. 5, cité par Foucault Michel, Surveiller et punir, Paris, Gallimard, 1975, p. 277. 
[...] Je me rappelle quà mon arrivée ici, pendant une de ces brillantes opérations d'encouragement à la réinsertion sociale, le détenu avait encore la permission de répondre, pour que tout le monde en ait le cour net: "Oui, monsieur, le connard a compris" ". ${ }^{12}$ Derrière ces violences carcérales, le protagoniste de Face au mur ouvre une brèche sur son propre passé de terroriste italien. Le lecteur peut alors songer aux argumentations fournies contre l'extradition des militants politiques italiens des années 1970 par Paolo Cento : "Invece di parlare di estradizione, l'Italia dovrebbe domandarsi perché il Brasile e la Francia vedono in maniera cosi negativa il nostro sistema giudiziario e carcerario $" .{ }^{13}$ On retrouve cette problématique aussi au-delà des Alpes. Bien que les juges français se soient opposés, en particulier, à l'extradition de Cesare Battisti pour une raison technique, le mythe d'un " asile politique " accordé à Cesare Battisti va prendre forme et Guillaume Perrault se voit obligé de commenter, après avoir précisé les motivations de l'opposition des juges français à l'extradition du terroriste italien : "Les avocats et les sympathisants de Battisti répéteront pendant des années que ce véto (opposition à l'extradition) des juges français traduit leur méfiance envers la justice de nos voisins $" .{ }^{14} \mathrm{Si}$ l'affaire Battisti est compliquée à démêler, il n'en reste pas moins que le 12 mars 2012, Vittorio Buffa, journaliste de L'Espresso, a dénoncé dans un dossier publié dans l'hebdomadaire italien, les sévices subis en prison par les terroristes italiens, pendant les années de plomb, lors des interrogatoires de la part de la police ${ }^{15}$. Face au mur nous oblige à nous plonger dans le passé historique tout en réfléchissant sur l'institut carcéral à notre époque. Le lecteur, à l'instar d'Auguste, navigue lui aussi entre l'Italie et le Brésil, entre le passé et le présent.

Le mur et l'enfermement constituent une thématique omniprésente dans ce roman. Le mot " mur " revient dans chaque chapitre, presque à chaque page, mais l'expression la plus fréquente est " au pied du mur " qui pourrait remplacer, par sa récurrence dans le récit, le titre même Face au mur, comme pour avouer une âpre défaite. Le terme " mur " tisse une trame tout au long du récit. L'enfermement, alors, se transforme en structure portante de l'œuvre tout entière.

Néanmoins, au-delà de la signification de l'emprisonnement, le mur représente surtout l'incommunicabilité des hommes au sein de la société, une société cloisonnée par différents remparts. Les paroles de Roberto Saviano viennent éclairer justement cet aspect souligné maintes fois par le protagoniste

12 Face au mur, op. cit., p. 119-121.

13 Cramer Francesco, « È sbagliato andare a caccia di ex terroristi », interview de Paolo Cento par Francesco Cramer, Il Giornale, 28/01/2009. "Plutôt que de parler d'extradition, l'Italie devrait se demander pourquoi le Brésil et la France ont une opinion si négative de notre système judiciaire et carcéral». Traduction par nos soins.

14 Perrault Guillaume, Génération Battisti : Ils ne voulaient pas savoir, Paris, Plon, 2005, p. 52.

15 Vittorio Pier, Buffa, "Torture di Stato ", L'Espresso, 12/03/2012, p. 70-72. 
du roman Face au mur: "Non avevo mai pensato a quanto fosse interessante parlare di muri. Non avevo mai pensato a come proprio il muro possa essere la chiave, per capire quanto sia piu facile allontanare da sé le proprie paure piuttosto che provare a risolverle. Quanto sia più facile marginalizzare una parte del paese e della società piuttosto che comprendere che è li, nella ferita, che il cuore pulsa. E se il cuore è malato, e non si pone rimedio, il paese va a fondo ". ${ }^{16}$

Les réflexions de Roberto Saviano sur la sémantique des murs qui enferment de différentes façons notre société semblent s'accorder avec celles du protagoniste, Auguste. Il faut dire que Saviano, tout comme Auguste, étudie les cloisonnements de la société en proie aux différents pouvoirs. ${ }^{17}$ Le mur est donc, dans le roman Face au mur la limite où se pose le regard d'Auguste, mais aussi l'appui pour prendre l'envol dans le récit. Le mur acquiert le statut de matrix dans laquelle le récit puise ses sources.

Le premier détenu décrit permet au protagoniste de présenter le personnage féminin le plus important du roman. Ainsi pourrions-nous d'abord étudier les caractéristiques de quelques femmes qui peuplent les pages de Face au mur.

Le premier portrait qu'Auguste présente au lecteur est celui de Méningite, qui demande à Auguste de lui révéler la signification des Écritures. Méningite, comme son sobriquet l'indique, ne brille pas par son intelligence. Souvent la plume du narrateur ne s'attarde pas sur l'aspect physique des personnages mais, en revanche, elle s'acharne toujours pour saisir l'aspect le plus pathétiquement humain de leur personnalité.

Méningite sert à introduire un des personnages-clefs du récit : Janaïna. En effet, l'évocation d'un des fléaux décrits dans la Bible, celui des insectes migrateurs dévorateurs de cultures, permet à Auguste de se souvenir de Janaïna, femme destructrice. Janaïna est l'élément dévastateur, elle est la locuste évoquée par Méningite.

En se servant toujours de cette technique des analogies et des contrastes qui nous fait penser, on l'a dit, à l'écriture cinématographique, Auguste substitue au portrait de Méningite celui de Janaïna, la femme qui, en obéissant

16 Saviano Roberto, "Lasciate tranquilli i bambini di Scampia ", L’Espresso, n 53, 3/01/2013, p. 7.

"Je n'avais jamais réfléchi à quel point c’était intéressant de parler de murs. Je n'avais jamais pensé au fait que justement un mur pût être la clef pour comprendre ô combien il est plus facile d'éloigner de soi ses propres peurs plutôt que d'essayer de les affronter et à quel point il est plus facile de marginaliser une partie du pays et de la société, plutôt que de comprendre que c'est là, dans cette blessure, que le cour bat et que si le cour est malade et qu'aucun remède n'est trouvé le pays sombre. "

17 Il existe un lien entre l'affaire Battisti et Roberto Saviano. Ce dernier avait signé, sur le site d'une revue on line, Carmilla, en février 2004, une pétition de soutien pour la libération de Cesare Battisti, arrêté en France le 10 février 2004. Saviano a demandé, par la suite, que sa signature soit ôtée de cette liste. Cf. Serra Elvira, "Saviano, Pincio e i pentiti dell'appello pro Battisti ", Corriere della Sera, 25/01/2009. 
aux services secrets brésiliens, partageait sa vie tout en exerçant un contrôle sur lui. C'est l'allégorie de la locuste de la Bible, à propos de laquelle Méningite demande une explication, qui rappelle à l'esprit du protagoniste le personnage de Janaïna : femme mi-déesse, mi-animal qui se dit amoureuse et en même temps l'empoisonne avec la complicité des agents des services secrets en charge du terroriste italien. Comment ne pas penser à Locuste, la célèbre empoisonneuse romaine évoquée par Suétone dans son ouvrage Vie des Douze Césars et par Tacite, dans ses Annales 18 $^{18}$

Le récit d'Auguste qui semblait jaillir d'une extrême spontanéité et se construire au gré des rencontres à l'heure de la promenade, dans la cour de la prison, répond, en revanche, à une logique bien construite de correspondances et puise ses sources dans la littérature et dans l'Histoire. À travers ces références littéraires et historiques, nous l'avons déjà souligné, le protagoniste de Face au mur (et de par là son auteur) cherche un statut et semble vouloir se frayer un chemin parmi les intellectuels français, dont il a élu la langue en récusant l'italien, afin, peut-être, de recevoir leur assentiment, leur approbation, voire leur protection. Il est désormais un apatride à la recherche d'une terre idéale, c'est pourquoi le choix de la langue française correspondrait à une véritable élection. Auguste semble porteur d'une vérité sur la société et choisit la nation qui pourra la recevoir, la France, la patrie des Droits de l'homme.

Écarter cette lecture c'est mésestimer la valeur symbolique de la culture française pour les Italiens.

La locuste est un insecte destructeur qui détermine une désertification après son passage. Auguste semble vouloir introduire cette dimension dans son récit quand il fait dire à Méningite : "J’y suis! [...] Ce n’est pas une question de langue, sinon tu me l'aurais tout de suite dit. Il s'agit d'une allégorie, c'est fréquent dans les Écritures de représenter le Mal sous la figure d'un être aux apparences bestiales qu'un bon chrétien risque de céder à la tentation rien qu'en s'y intéressant ". ${ }^{19}$ Janaïna est la tentation, elle est très jeune, belle et fort sensuelle. Après son passage, il ne reste que dévastation dans la vie d'Auguste. Janaïna possède toutes les qualités et tous les vices, mais sa nature est essentiellement diabolique. Elle fait partie de cette galerie de portraits féminins qui illustrent la duplicité de la femme, tantôt mère, tantôt prostituée. Presque toutes les femmes du roman Face au mur sont des personnages calculateurs, infidèles, douteux. Si la société est partagée entre le Bien et le Mal, les personnages féminins, eux, se tournent, pour la plupart, indubitablement vers la perversion.

Aurea, la femme fatale, qui attrape Auguste dans ses filets faits de délices et de ruses et le dirige savamment vers le Brésil, n’a du métal dont elle porte le nom que l'éclat doré d'une intelligence hors du commun, mais pernicieuse :

18 Suétone, Vie des douze Césars, Néron. Tacite, Annales, livre XIII.

19 Face au mur, op. cit., p. 22. 
"Aurea. C'est drôle, je crois que son rêve avait d'abord été d'être une bonne sour. Mais elle a fini par devenir une pute internationale ${ }^{20}$ Elle est, elle aussi, à la solde des services secrets.

Sandra, médecin philanthrope d'origine française, animée par des rêves de justice sociale, aurait pu être un personnage féminin positif si elle n'avait pas fini par noyer ses désillusions dans l'alcool et le "xango ", une sorte de culte introduit au Brésil par les esclaves africains. La seule femme qui semble racheter ces personnages féminins à la noirceur troublante est Ester, "dona Ester" qui vit dans le "morro $"^{21}$. Son fils Jonas n'avait que douze ans et il est mort de "trois balles dans la poitrine et une dans la gorge. Une faction rivale avait tenté d'envahir sa "boca"22 à l'aube ". ${ }^{23}$ Ester, pour vivre, a besoin de médicaments coûteux et son fils se procurait l'argent pour maintenir en vie sa mère en étant un "soldat " de la drogue. Cette femme pleure la mort de son fils dans une espèce de sanctuaire domestique dédié à Saint Georges et désormais à l'enfant disparu. Néanmoins, il s'agit d'un personnage féminin écarté du contexte social car elle a toujours vécu dans une sorte de ségrégation. Elle est, certes, la mater dolorosa, mais aussi celle qui a accepté que son fils, un enfant de douze ans, sacrifie sa vie en échange de la sienne.

L'autre personnage féminin qui semble appartenir à la sphère du bien, selon un partage dichotomique et manichéen, est Larissa. Vêtue de son innocence et de sa candeur, cette jeune femme cherche seulement à récupérer un objet ayant appartenu à son frère après son meurtre. Elle se retrouvera, à son insu, impliquée dans une sordide affaire de drogue et se pendra en prison, avant son procès. Son histoire, qui relève de l'hagiographie, semble aspirer à montrer les méandres hideux d'une société corrompue et corruptrice. Le paradigme du narrateur réside dans la certitude que la société en elle-même porte le germe de la discorde, de la perversion, voire de l'immoralité pure.

Les rues des villes brésiliennes, parcourues par Auguste, dans ses souvenirs d'homme libre, sont peuplées d'un flot de gens qui ne semblent pas avoir de visage pour le protagoniste. On dirait que ces villes sont habitées par des ombres.

Les seuls portraits pluridimensionnels, dans ce roman, sont ceux des prisonniers du pénitencier de Brasilia, comme si seul l'enfermement réussissait à révéler la vraie essence de l'homme qui arrive à se connaître dans l'autre : "Se refléter dans les yeux des autres ou sonder son état d'àme en se tâtant les plis de la peau, cela peut t'aider à faire le rapprochement, je dirais même l'union, entre le corps et l'esprit ${ }^{24}$

20 Face au mur, op. cit., p. 167.

21 Dans le contexte de Rio, référence aux quartiers pauvres qui sont situés sur les nombreuses montagnes de la ville.

22 "Bouche de fumette », point de vente de la drogue.

23 Face au mur, op. cit., p. 159.

24 Ibid., p. 13. 
Zeca est l'un des détenus de la prison où se trouve le protagoniste. Zeca est fasciné par la ville de Rio, capable d'envolées lyriques face aux photos de cette ville. Zeca est un tueur à gage et ne connaît qu'une force, celle des armes. Sa morale consiste à promettre qu'il ira mettre une balle dans la tête aux dealeurs qui vendent de la drogue mal coupée, dès sa sortie de prison.

Les détenus décrits par Auguste ont souvent une aspiration qui loin d'être morale, est tout de même ressentie comme telle.

L'histoire de Bruno en est le meilleur exemple. Bruno revient dans son Amazonie natale après avoir terminé ses études. Entre réalité, rêves et croyances ancestrales, l'histoire de ce jeune homme se dessine comme un songe à l'allure cauchemardesque. Le lecteur ne sait jamais si la réalité est bien celle qui est relatée, car le narrateur le met en garde sur la confusion qui règne dans l'esprit de Bruno : "[...] la situation lui était devenue trop lourde à supporter. Même pour lui qui essayait de s'accrocher à la science, à la raison, et à un matérialisme, je dirais, obstiné, lui dont la tête était farcie de lectures au point de n'être plus capable de distinguer les émotions de la raison, lui dont l'équilibre était déjà mis à rude épreuve à chaque prise excessive de médicaments $"{ }^{25}$

Le personnage de Bruno est celui du justicier que rien ne prédisposait à cette tâche sinon un esprit voué à la pureté et à l'ordre. Son histoire occupe un chapitre entier et c'est avec ses vicissitudes que la galerie de portraits des détenus s'achève. C'est ainsi que Bruno représente une sublimation des vertus de tous les autres prisonniers, en étant capable de tuer pour libérer son peuple des supercheries d'un personnage équivoque. Il s'agit d'un représentant de l'État qui néanmoins acquiert une dimension mythique et porteuse de détresses pour les gens du village. L'allégorie de Bruno est celle de l'homme honnête qui devient meurtrier face à un pouvoir prévaricateur. Le roman semble donc une démonstration logique qu'une société corrompue engendre nécessairement des justiciers qui enfreignent la loi au nom du désir ardent d'un principe moral supérieur. Ce concept soutient tout le roman qui est parsemé de phrases qui font allusion tantôt à la nécessité de la justice et du changement, tantôt aux idéaux révolutionnaires : "À ce moment, j'ai su que ma raison d'être au Brésil venait de l'exigence impérieuse de reconstruire l'un après l'autre les ponts que j’avais jadis brûlés derrière moi, en une retraite ordonnée, digne de ces grands idéaux capables de transformer l'esprit révolté en chair à canon. $»^{26}$; "D'un autre côté, l'Italie et l'Europe, qu'auraient-elles en échange? Un décombre des années 1970, un petit rêveur à l'époque et un vieux con de rêveur aujourd'hui. " ${ }^{27}$; "Ce n'était pas la première fois que je me souvenais [...]. Bien des années après [...], alors que jétais pris dans les habits trop

25 Ibid., p. 255.

26 Ibid., p. 68.

27 Ibid., p. 108. 
serrés du "soldat de la libertê". ${ }^{28} ;$ " En quelque sorte, mon arrestation má évité de toucher le fond, d'être rattrapé par mes anciennes frustrations au sujet d'une société en pleine dégénérescence, société que j'avais rêvé de changer. " ${ }^{29}$; "À l'abri du bien et du mal, je reconstituais les événements qui avaient transfiguré la vie d'une génération qui avait osé universaliser la révolte. " ${ }^{30}$

Le personnage de Bruno acquiert alors une autre signification. Il devient le pendant d'Auguste. Ce dernier a cru, dans sa jeunesse, qu'il était appelé à un possible rachat de la société italienne par des actions violentes. À présent, Auguste justifie ses choix, plaidant non coupable dans la mesure où les "grands idéaux " sont "capables de transformer l'esprit révoltés en chair à canon"! D'ailleurs il n'est plus qu'un "vieux con de rêveur"; peut-on avoir peur d'un rêveur et de surcroît "vieux » et "con » ? En outre, on dirait que la liberté elle-même oblige l'homme à vêtir ses " habits trop serrés de soldat " et donc cette dernière excuserait tout acte violent accompli en son nom. Auguste se plaçant au-delà du "bien et du mal ", tel un être métaphysique, constate, enfin, que la vie d'une génération qui a "osé universaliser la révolte " est désormais " transfigurée " d'où ce participe passé qui, dans son contexte, acquiert une acception négative et presque sinistre. Peut-on demander une punition plus sévère pour toute une génération, semble-t-il nous demander? Le roman Face au mur, là où il cherche des éléments à décharge des accusés du terrorisme des années 1970, acquiert, sans aucun doute, une dimension politique, savamment dissimulée. C'est ainsi que ce roman s'inscrit dans une volonté de réflexion autobiographique et constitue une tentative, sinon d'un plaidoyer, au moins d'une excusatio pour toute une génération dévastée par la violence du terrorisme. La fiction romanesque a une visée politique.

\section{L'écriture entre dénégation et construction}

Le personnage de Bruno est sans aucun doute le plus emblématique par son déni de l'acte délictueux. L'article de Freud qui décrit le mécanisme que la conscience adopte, dans certains cas, afin de se défendre par une négation des pensées et/ou des actes qui ne peuvent pas être assumés porte le nom de dénégation. Sous cette appellation on retrouve ce concept dans les travaux de psychologie et de psychiatrie de plusieurs auteurs.

Ce procédé concerne les réactions de la conscience face à des événements traumatisants que cette dernière doit affronter. Dans son article de 1925, Freud écrit : "La tâche de la fonction intellectuelle de jugement étant d'affirmer ou de nier des contenus de pensée, [...]. Nier quelque chose dans le jugement veut 
dire au fond: c'est là quelque chose que je préférerais de beaucoup refouler. ${ }^{31} \mathrm{La}$ dénégation est un processus de défense auquel la conscience recourt lorsqu' elle n'arrive pas à intégrer un fait traumatisant. ${ }^{32}$

Auguste décrit toujours des événements tragiques lorsqu'il peint les vies de ses compagnons d'infortune. Sa plume doit s'adapter à décrire la violence, les méfaits, les délits. Le ton du roman est indubitablement, surtout, celui de la tragédie. Du moment où le meurtre s'accomplit, l'écriture devient néanmoins lacunaire, avare d'informations, capable de se dissiper dans un brouillard de suppositions où le lecteur souvent s'égare. On retrouve Bruno en prison mais le doute sur sa culpabilité persiste. S'agit-il d'un refus de reconnaître la réalité d'un acte traumatisant de la part de ce personnage, ou alors, d'un abus d'un pouvoir corrompu l'ayant incarcéré illégalement?

La responsable de cette ambiguïté est l'écriture. Ainsi en sera-t-il pour le personnage d'Ińacio à propos duquel le protagoniste formule, tout de même, quelques doutes, quant à sa volonté d'avouer son crime : «À ce point de l'histoire, la suite des événements vécu par Iñacio devient moins claire. Mon impression est qu'Iñacio aurait bien voulu en gommer une partie. "33 $^{33}$

L'écriture s'évertue à esquiver ces moments de description des faits délictueux, comme si elle devenait " aphasique ». Le protagoniste, parfois, interprète les faits afin de justifier les agissements des détenus. Freud écrit dans son article sur la dénégation : "La reproduction de la perception dans la représentation n’en est pas toujours la répétition fidèle; elle peut être modifiée par des omissions altérée par des fusions entre divers éléments. »" Auguste applique la même technique descriptive aussi à ses propres souvenirs relatant la période de son appartenance à la lutte armée, dans une patente volonté d'égarer le lecteur entre réalité et interprétation de cette dernière. Cette écriture parfois lacunaire et dépouillée, parsemée d'omissions et de flottements entre le rêve et la réalité, est celle de la dénégation.

Auguste possède un pouvoir que les autres détenus n’ont pas : la puissance de l'écriture. Tous les prisonniers du pénitencier de Brasilia savent qu'Auguste confie leurs histoires à un "cahier jaune ". Les détenus ont donc une seconde possibilité par rapport à leur culpabilité qui consiste à relater à Auguste leur

31 Freud Sigmund, Résultats, idées, problèmes II, 1921-1938, Paris, Presses Universitaires de France, 1985, p. 136.

32 L'explication fournie par le manuel de psychologie peut éclairer ce mécanisme de la psyché : "La dénégation est un désaveu direct des données sensorielles traumatisantes [...]; d'habitude la dénégation est une défense contre une réalité du monde extérieur, lorsque cette dernière est ressentie comme étant terriblement dérangeante. Bien que ce mécanisme soit souvent associé à des psychoses et à des troubles graves de la personnalité, il peut être présent aussi chez des personnes normales, surtout ayant fait face à des événements catastrophiques. "Gabbard Glenn O. , Psichiatria e psicodinamica, Milan, Raffaello Cortina Éditeur, 1992, p. 46. Traduction par nos soins.

33 Face au mur, op. cit., p. 319.

34 Freud Sigmund, Résultats, idées, problème II, 1921-1938, op. cit., p. 138. 
version des faits. Ainsi Auguste devient-il un démiurge à la plume créatrice de réalités acceptables. Auguste, le célèbre terroriste italien arrivé dans la prison escorté incroyablement d'un seul agent de police pénitentiaire, Auguste à l'air inoffensif, Auguste, le solitaire et le pensif, acquiert une force inouïe, celle de l'écriture. Tous les détenus le respectent pour son "cahier jaune " et même les criminels les plus endurcis savent reconnaître la puissance de l'écriture, capable d'ériger des mondes et avec eux des vérités.

Pourtant Auguste doute, parfois, de son pouvoir : "Au moment où j’écris, je me demande si j'ai le droit de fouiller ainsi dans sa vie. J'ai peur de lui faire tort. ${ }^{35}$

Les détenus se présentent alors à Auguste pour susciter son intérêt afin que ce dernier raconte leur histoire. Tels qu'Ersilia, dans la pièce de Pirandello Vêtir ceux qui sont nus, les prisonniers habillent leur vie de vêtements dignes d'être "vus ». Chacun, comme l'Ersilia de Pirandello, est une âme nue et ressent la nécessité de se draper dans une certaine respectabilité avant de se présenter à autrui. Le "cahier jaune " d'Auguste serait la preuve que seuls la littérature et l'art sont à même de rendre compte de la souillure indélébile laissée sur eux par l'emprisonnement. Il démontre aussi que seul l'imaginaire peut les vêtir décemment.

Exercice périlleux que celui d'habiller ceux qui sont " nus ». Auguste fait dire à Bruno: "Je te parle de moi, de ma terre, de ma vie, considère cela comme un cadeau et tu t'en tiens là. S'ouvrir à l'autre, c'est traverser l'enfer sans Virgile, ne l'oublie pas. " ${ }^{36}$ Néanmoins, ce n'est pas Virgile, mais Dante lui-même qui, en traversant les horreurs de l'Enfer, adopte une langue capable de s'adapter à la " comédie " humaine, tout comme le protagoniste de Face au mur qui jongle entre plusieurs registres de langue afin de permettre à cette humanité déchue de s'exprimer. D'ailleurs, en préface du roman, on lit une citation de Dante, comme pour invoquer non pas une Muse, mais le Poète lui-même, afin d'inscrire cet iter littéraire, idéalement, dans son sillon, à la recherche non pas de la vérité mais plutôt du courage nécessaire pour l'affronter.

C'est alors, à travers ce projet d'écriture, qu'Auguste comprend qu'écrire sur les autres équivaut à écrire sur soi-même : "Au moment où j'écris, je me demande si j'ai le droit [...]. Mais j'ai aussi l'impression d'explorer des régions inconnues de moi-même, poussé par ce que je me sens de commun avec Bruno, notre incapacité à vivre le bonheur. ${ }^{37}$; "Soyons honnête, je les écoute surtout parce qu'il y a tant de maladresse dans leur vie que je m'y reconnais. Et cela me fait du bien de nêtre pas le seul. ${ }^{38}$ Face au mur devient donc un vaste domaine d'introspection où Auguste, dans la parole de ses compagnons de captivité,

35 Face au mur, op. cit., p. 221.

36 Ibid., p. 256.

37 Ibid., p. 221.

38 Ibid., p. 338. 
peut se reconnaître. C'est alors que le verbe des détenus, dans ce récit, acquiert la mobilité inquiétante des eaux, où Auguste, tel un Narcisse, se contemple et parfois arrive à s'aimer dans la fuite de ce flux de mots.

Cet exercice devient vite risqué et Auguste se reconnaît "et s'aliène dans un reflet qui lui révèle [...] son illusoire et fugace existence : toute sa vérité dans un fantôme, une ombre, un rêve " ${ }^{39}$ dans cette réalité in fieri façonnée par la parole d'autrui et de cela naissent ses retraites solitaires " face au mur ». Sans doute l'écriture représente-t-elle un substitut, un Ersatz, à une psychanalyse qui s'ébauche pour le protagoniste, dans la découverte de sa propre personnalité dans le regard de l'autre et l'autre est souvent un détenu.

L'écriture a comme ambition un projet de reconstruction du moi. Auguste est un grand bâtisseur de... ponts : "reconstruire un après l'autre les ponts que j’avais jadis brûlés ", ${ }^{40}$ promet-il au lecteur. Néanmoins, les travées de ses " ponts " vont s'étendre, non pas entre le passé et le présent, mais plutôt entre la réalité des faits et sa dénégation, tout en constituant, et le protagoniste en est certain, "l'affaire Auguste $"{ }^{41}$. Tout au long du roman, il donne de lui l'image d'un homme inerme et pacifiste en pleine contradiction avec le personnage que les gouvernements ont créé. "Les médias avaient donné de moi l'image d'un "irréductible" (sic). " ${ }^{42}$ Son arrestation est pour Auguste une "opération politique rentable " ${ }^{43}$. Sa fuite de la France est interprétée comme une violence subie : "Cruel ne rate pas une nouvelle murmurée dans le bloc ainsi que celles qui passent à la télé, et il sait que jaime analyser les événements révélateurs d'un pays dont on m'a arraché avant que j'aie eu le temps de comprendre. " ${ }^{44}$ Les traces de cette dénégation de culpabilité sont de plus en plus évidentes et nombreuses, au fur et à mesure que coule l'encre d'Auguste, ce qui nous porte à dire que tout le roman est une excusatio, un vaste plaidoyer construit savamment plein de renvois qui se répondent et s'interpellent à l'intérieur d'une écriture à architecture solide.

C'est ainsi que le protagoniste, à la fin de son récit, dans un souci de symétrie, retourne à l'observation de son petit oiseau qui avait constitué l'incipit de l'œuvre : "Le ciel n'est plus qu'une intuition d'espace et le petit oiseau blanc est allé se coucher. Sur l'antenne ou sur l'eucalyptus? Rio ou Brasilia? J'aimerais savoir où il a choisi son nid ce soir, pour le suivre bientôt dans son sommeil. " ${ }^{45}$

39 Genette Gérard, Figures I, Paris, Éditions du Seuil, 1966. p. 25.

40 Face au mur, op. cit., p. 68.

41 Ibid., p. 349.

42 Ibid., p. 269.

43 Ibid., p. 268.

44 Ibid., p. 103.

45 Ibid., p. 338. 


\section{Considérations conclusives}

Le dernier roman de Cesare Battisti retrace son histoire personnelle, il en est une interprétation littéraire. De grands pans de son passé de militant de la lutte armée sortent ainsi des pages de cet ouvrage. La thématique du mur et de l'emprisonnement soutiennent l'édifice de cette œuvre qui puise à pleines mains dans la littérature, plutôt que dans une prétendue spontanéité. L'auteur propose une réflexion, à travers ces pages, sur la responsabilité de la société elle-même dans les délits qui sont commis et sur la punition infligée par l'enfermement qui isole les hommes, les pervertit et crée l'aliénation de l'individu. Néanmoins, ces pages connaissent des moments d'élégie dans la description de cette poignante humanité qui anime presque tous les détenus de cette frange oubliée de la société. La description des différents personnages, qu'ils soient masculins ou féminins, constitue une vaste fresque de cette société sud-américaine. On pourrait presque croire que les pages de Face au mur représentent une possibilité d'étudier cette étrange et disparate communauté carcérale ou de trouver les causes ultimes, l'étiologie presque de la perversion si enracinée chez les héroïnes de ce roman. On se tromperait, car Face au mur ne présente qu'un seul et unique personnage : Auguste qui s'entoure d'un immense chœur tout en demeurant maitre absolu et incontesté de la scène, sa scène où il achève sa tragédie.

Ce roman a été écrit en langue française car Cesare Battisti lui-même explique, dans son interview avec Le Point du 17 février 2012, qu'il a cessé d'écrire en italien, en réponse à l'attitude des hommes politiques italiens l'ayant "massacré d'une façon immonde ». Rejeter la langue italienne signifie pour Battisti se déclarer, en quelque sorte, apatride et donc subrepticement en droit d'une protection internationale. Cesare Battisti, en adoptant la langue française, cherche alors une appartenance idéale et tente de s'insérer dans le cercle très ambitionné des intellectuels français. La réflexion sur la période des années de plomb, en outre, fait de Face au mur, défini " roman " par les éditions Flammarion, une sorte d'essai et par là, cet ouvrage s'enrichit d'une visée fortement politique.

Cependant, dans le rejet de sa langue maternelle, nous y voyons aussi une volonté, bien qu'inconsciente, de refouler son passé, et presque de le nier. L'écriture de Battisti, par moments amnésique et lacunaire, naît d'un désir de façonner les événements afin de les apprivoiser. C'est ainsi qu'entre réalités douloureuses et dénégations, par-delà tout enfermement physique et toute réclusion de l'esprit, il existe une ressource, semble dire Cesare Battisti, qui est l'écriture, la seule activité qui de par sa vertu créatrice et son souffle libérateur arrive à reconstruire l'âme de tout homme. 